\title{
PRINCIPALES CARACTERISTICAS DE LA POBLACION DE LA VILLA DE MADRID POR DISTRITOS MUNICIPALES
}

\author{
$311: 352$ (46, Madrid)
}

por

\section{Ignacio Ballester Ros}

SUMARIO: I. INTRODUCCION.-II. DISTRIBUCION DE LA POBLACION: 1. EN CONJUNTO. 2. POR SEXo. 3. POR EDAD.-III. EL LUGAR DE NACIMIENTO DE LA POBLACION DE MADRID: 1. EN LINEAS GeNerales. 2. Por RegIONES DE PROCEDENCIA.-IV. LA CLASE DE ENSENAANZA MAS ELEVADA RECIBIDA POR SUS HABITANTES.V. CARACTERISTICAS DE LA POBLACION ACTIVA: 1. LA PROFESIÓN. 2. La SITUACIÓN PROFESIONAL. 3. La RAMA DE ACTIVIDAD ECONÓMICA.

\section{INTRODUCCION}

El Instituto Nacional de Estadística ha tratado de atender las recomendaciones de la Confederación de Estadísticos Europeos acerca de la conveniencia de llevar a cabo investigaciones de la población en los períodos quinquenales intermedios entre los grandes censos decenales de población. Y para ello ha aprovechado el Padrón Municipal de Habitantes, documento de carácter público en orden a la existencia y características de los habitantes, de inapreciable valor demográfico, cuya renovación quinquenal y rectificaciones anuales realizan los Municipios españoles.

El Instituto realizó por primera vez en 1965 un ensayo para obtener, con fines estadísticos, una explotación de los datos de la renovación padronal, a partir de una muestra del 25 por 100 de las familias empadronadas; y los resultados se publicaron en una monografía titulada Estadisticas de la población de España, deducidas del Padrón Municipal de Habitantes del año 1965. 
De igual manera, el Instituto ha repetido el experimento en 1975, si bien con mayor amplitud, tomando como base la inscripción padronal referida al 31 de diciembre de dicho año, mediante una muestra algo más reducida del 20 por 100 de las familias registradas; y sus resultados se han publicado en doce volúmenes, bajo la denominación genérica de Características de la población española, deducidas del Padrón Municipal de Habitantes, según la inscripción realizada el 31 de diciembre de 1975. En esta serie monográfica se presentan los resultados obtenidos de carácter nacional y provincial, y los relativos a las capitales de Provincia y a los Municipios mayores de 50.000 habitantes individualmente considerados.

Esta información pretende ser, de alguna manera, una continuación de la suministrada por el Censo de Población de 1970, si bien menos ambiciosa, dada la limitación que lógicamente existe en los datos primarios. $Y$ sirve para atender, en gran parte, la creciente demanda de información referente a las características de la población.

En la última publicación citada hay una importante novedad, ya que, con referencia a los Municipios de Madrid y de Barcelona, se presentan los resultados obtenidos por distritos municipales.

Las características que se analizan con referencia a cada distrito son: la población de derecho y de hecho, el sexo, la forma de vivir en familia o no, la edad, el estado civil, el lugar de nacimiento de la población española - por Provincias de procedencia- y la clase de enseñanza más elevada recibida.

Una atención especial se dedica a la población económicamente activa, de la que se estudia la profesión, la situación profesional, la rama de actividad económica y la condición socioeconómica.

Dada la riqueza informativa enumerada, limitamos este comentario al estudio de la población de Madrid por distritos municipales, en relación con algunas de las características de mayor significación.

Ya a tal fin hemos reelaborado los resultados expresados en cifras absolutas, presentándolos sistemáticamente en porcentajes para facilitar su examen comparativo.

\section{DISTRIBUCION DE LA POBLACION}

Como es sabido, Madrid está dividido en la actualidad en dieciocho distritos municipales, numerados en forma de dos círculos 
concéntricos, más reducido territorialmente el interior y más amplio el exterior o periférico.

En la tabla I del apéndice estadístico se ofrece la distribución, por distritos municipales, de los habitantes, según sexo y edad.

\section{EN CONJUNTO}

El promedio teórico de la población de cada distrito municipal de Madrid es del 5,5 por 100 del total. Se advierten, sin embargo, notorias diferencias en su cuantía entre los distintos distritos, pudiendo formar con ellos dos grupos: el primero, de ocho distritos, cuya población supera el promedio municipal, y el segundo, con los diez distritos restantes.

Los distritos del primer grupo configuran, a su vez, dos zonas: la primera está constituida por los distritos Centro, Chamberí y Salamanca, distritos clásicos de la villa, que se ubican en el casco antiguo y en el viejo ensanche; y la segunda, formada por los distritos de Ciudad Lineal y Vallecas, al este de la avenida de la Paz, y los de Latina, Carabanchel y Villaverde, al sur del río Manzanares, claramente periféricos todos ellos. Los más poblados son Latina, con su contiguo Carabanchel, y Ciudad Lineal.

Entre los del segundo grupo podemos igualmente formar una amplia zona de distritos periféricos: Moncloa, al oeste; Fuencarral, al norte; Hortaleza, San Blas y Moratalaz, al este, y Mediodía; y otra zona interior, con Arganzuela y Retiro, Tetuán y Chamartín. Los menos populosos de entre ellos son Moncloa, Retiro y Arganzuela.

La desigual proporción en la población de los distritos depende, en gran parte, de sus respectivos límites administrativos; pero se puede afirmar, sin embargo, que los siete distritos del círculo concéntrico interior - Centro, Arganzuela, Retiro, Salamanca, Chamartín, Tetuán y Chamberí- albergan sólo a algo más de la tercera parte de los habitantes de la villa, mientras que casi los dos tercios de la población de Madrid residen en el círculo concéntrico exterior de distritos periféricos, constituido por los once distritos restantes.

\section{POR SEXO}

Como término medio, existen 108 mujeres por cada 100 hombres en Madrid; pero se registra una patente desigualdad entre los 
distritos. De una parte, se hallan ocho distritos: Centro, Arganzuela, Retiro, Salamanca, Chamberí, Tetuán y Chamartín, todos del círculo interior, y Moncloa, con un promedio de mujeres por 100 hombres superior al promedio municipal, destacando entre ellos Salamanca, con 125, y Centro y Chamberí, con 122 mujeres por cada 100 hombres. De otra parte, se hallan todos los demás distritos, periféricos todos ellos, con valores bastante inferiores al indicado promedio, y aun dos de ellos, Latina y Mediodía, tienen más hombres que mujeres.

Existe, pues, una desigual composición de la población por sexo, advirtiéndose una menor proporción de mujeres en los distritos de más reciente expansión demográfica, los clásicos de inmigración, y una proporción sensiblemente mayor de mujeres en los distritos antiguos, con población más asentada.

\section{POR EDAD}

En la distribución por grupos de edad de la tabla que analizamos se advierte que, en conjunto, de cada 100 habitantes tienen hasta veinticuatro años 42 ; entre veinticinco y sesenta y cuatro, 49, y solamente nueve rebasan los sesenta y cinco años. Se trata de una población progresiva, con mucha juventud y un porcentaje relativamente reducido de la denominada tercera edad.

Al examinar la distribución por distritos, se observa que la población joven reside con preferencia en los diez distritos de Chamartín, Fuencarral, Latina, Carabanchel, Villaverde, Mediodía, Vallecas, Moratalaz, Ciudad Lineal, San Blas y Hortaleza, todos ellos periféricos, con la excepción de Chamartín. Por el contrario, la tercera edad predomina en los siete distritos de Centro, Arganzuela, Retiro, Salamanca, Tetuán, Chamberí y Moncloa, correspondiendo los valores máximos a Centro, Salamanca y Chamberí.

La población en edad adulta presenta, en general, escasas diferencias alrededor del valor promedio del Municipio.

De las cifras anteriores puede deducirse la distribución de la población activa. Anotemos, aunque no se deduce de la tabla que comentamos, que la población activa femenina representa el 27 por 100 de la población activa total y se ubica con preferencia en los distritos Centro, Arganzuela, Retiro, Salamanca, Chamartín, Tetuán, Chamberí, Moncloa y Latina. Los restantes distritos aparecen con una población activa femenina inferior al promedio municipal. 


\section{EL LUGAR DE NACIMIENTO DE LA POBLACION DE MADRID}

\section{EN LINEAS GENERALES}

No se ha investigado en el trabajo que comentamos la población residente y nacida en la villa de Madrid, aunque sí se conoce la nacida en la Provincia de Madrid, que representa el 52 por 100 de la población del Municipio. De cada 100 habitantes, 47 han nacido en otras Provincias y uno en el extranjero.

En su distribución por distritos municipales, según la tabla II, se registran diez de ellos con una proporción de nacidos en la Provincia superior al promedio municipal. Son los de Centro, Retiro, Chamartín, Tetuán, Fuencarral, Latina, Moratalaz, Ciudad Lineal, San Blas y Hortaleza, indistintamente centrales o periféricos, siendo los dos últimos citados los que albergan a más nativos de la Provincia.

Correlativamente, son los ocho distritos restantes los que albergan a mayor número de habitantes nacidos en otras Provincias, en especial el de Arganzuela, único distrito con una proporción de nacidos en otras Provincias superior al de nacidos en la Provincia de Madrid.

Los habitantes nacidos en el extranjero se distribuyen por todos los distritos, aunque los de Salamanca y Chamartín son los que albergan a mayor número de ellos, y Villaverde y Mediodía los que menos tienen.

\section{Por Regiones DE PROCEDENCIA}

Madrid es el foco de atracción demográfica más importante de España, por la intensidad del movimiento inmigratorio y por la amplitud territorial a que se extiende su influencia, ya que ha recibido inmigrantes de todas las Regiones españolas.

Podemos, sin embargo, señalar la existencia de dos amplias zonas teritoriales de desigual aportación al movimiento inmigratorio de Madrid.

La primera está constituida por ambas Castillas, León, Extremadura y Andalucía - prácticamente la Meseta y Andalucía-, que aportan 81 de cada 100 inmigrantes. Y la segunda, por las restantes Regiones españolas, que, en conjunto, aportan 19 inmigrantes de cada 100. 
Las Regiones que configuran la primera zona aportan inmigrantes en la siguiente proporción:

\section{Porcentajes}

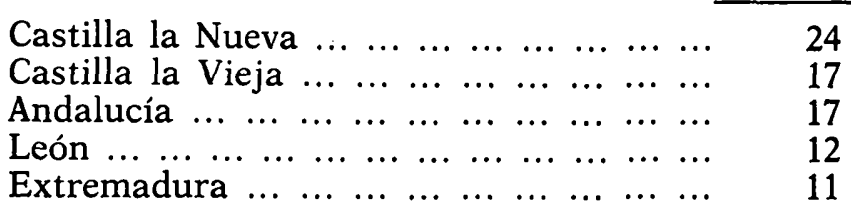

es decir, que la quinta parte de la inmigración procede de las cuatro Provincias restantes de Castilla la Nueva, mayoritariamente de las de Toledo y Ciudad Real. Castilla la Vieja, singularmente las Provincias de Avila y Segovia, y Andalucía, particularmente las Provincias de Córdoba y Jaén, aportan cada una la sexta parte de los inmigrantes madrileños. Todas las Provincias del antiguo Reino de León aportan contingentes parecidos, con la excepción de Palencia.

Pudiera afirmarse que la Submeseta Norte aporta la tercera parte de los inmigrantes, la Submeseta Sur otra tercera parte y Andalucía una sexta parte.

De la segunda zona, ocupa el primer lugar Galicia, que aporta el 4 por 100 de inmigrantes; Asturias, Murcia y VascongadasNavarra aportan el 3 por 100 cada una, y Valencia, CataluñaBaleares y Aragón, el 2 por 100 cada una. La aportación canaria es muy reducida.

Parece interesante conocer de qué manera se distribuye la población por distritos municipales en razón de las Regiones de procedencia. En la tabla III - porcentajes en línea- se mide la aportación de cada Región a la población de cada uno de los distritos municipales, tomando a éstos como unidad de distribución.

Los inmigrantes procedentes de Castilla la Nueva residen preferentemente en los distritos interiores de Centro y Arganzuela y en los periféricos de Latina, Carabanchel, Villaverde, Mediodía, Vallecas, Moratalaz y San Blas.

Los andaluces se ubican, sobre todo, en los distritos de Fuencarral, Moncloa, Mediodía, Vallecas, Moratalaz, San Blas y Hortaleza, todos ellos periféricos, salvo el primero.

Los inmigrantes procedentes de Castilla la Vieja, por una parte, en distritos interiores, como Centro, Salamanca, Chamartín, Tetuán, Chamberí y Fuencarral, y de otra, en los periféricos de Moncloa, Latina, Carabanchel, Ciudad Lineal y Hortaleza. 
Los leoneses se distribuyen en forma más amplia, residiendo con carácter de preferencia en doce distritos, todos los interiores y los periféricos de Moncloa, Latina, Carabanchel y Ciudad Lineal.

Los extremeños residen, por el contrario, en todos los periféricos, salvo Moncloa y Ciudad Lineal, y en el interior de Fuencarral.

Los gallegos, asturianos, murcianos, valencianos, catalanes, aragoneses $y$ vascos residen preferentemente en distritos interiores $\mathrm{y}$ en algunos de los periféricos, como Moncloa, Latina, Ciudad Lineal, San Blas u Hortaleza. Los canarios se ubican en áreas más restringidas, en especial los distritos de Arganzuela y Chamartín, Moncloa, Ciudad Lineal y Hortaleza.

La tabla IV - porcentajes en columna- nos permite conocer con mayor precisión la distribución de cada colectivo regional por distritos municipales, tomando como unidad de referencia a cada uno de estos colectivos.

En este sentido, los castellanos, leoneses, extremeños y andaluces se ubican predominantemente en los distritos periféricos de Latina, Carabanchel, Villaverde, Mediodía, Vallecas y Ciudad Lineal. Por el contrario, los gallegos, asturianos, murcianos, valencianos, catalanes, aragoneses, vascos y canarios residen sobre todo en los distritos de Centro, Salamanca, Chamberí, Chamartín, interiores, y Latina, Carabanchel y Ciudad Lineal, periféricos.

\section{LA CLASE DE ENSEÑANZA MAS ELEVADA RECIBIDA POR SUS HABITANTES}

Los habitantes de Madrid se distribuyen, en 1975, según el nivel de estudios alcanzado, en esta forma: de cada cien, poseen:

$\begin{array}{lllllllllll}\text { Estudios universitarios } & \ldots & \ldots & \ldots & \ldots & \ldots & \ldots & \ldots & \ldots & 7\end{array}$

$\begin{array}{llllllllllll}\text { De segundo ciclo } & \ldots & \ldots & \ldots & \ldots & \ldots & \ldots & \ldots & \ldots & \ldots & \ldots & 4\end{array}$

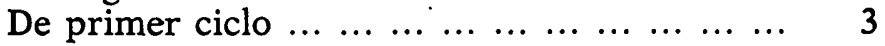

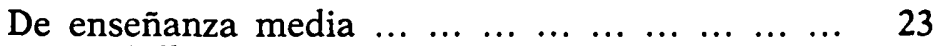

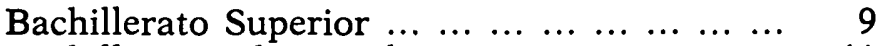

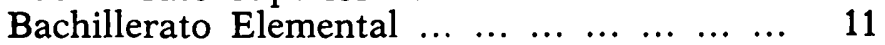

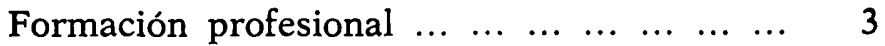

$\begin{array}{lllllllllll}\text { De enseñanza primaria } & \ldots & \ldots & \ldots & \ldots & \ldots & \ldots & \ldots & \ldots & 30\end{array}$

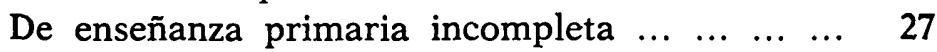

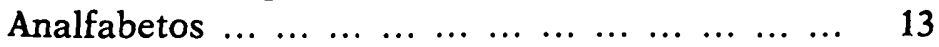

La distribución por residencia en los distintos distritos municipales es muy significativa, según se deduce de la tabla V. Los titu- 
lados superiores, procedentes de Facultades universitarias y Universidades politécnicas, residen preferentemente en ocho distritos, en especial en los de Salamanca, Chamartín y Chamberí; a segundo nivel, en los de Moncloa y Retiro, y con valores iguales o ligeramente superiores al promedio municipal, en Centro, Arganzuela y Tetuán, configurando una zona sin solución de continuidad, limitada al sur por el río Manzanares, al este por la avenida de la Paz y extendiéndose por el oeste hasta el término municipal de Pozuelo de Alarcón.

Los que poseen estudios universitarios de primer ciclo se distribuyen, según su residencia, en forma bastante similar a los del segundo ciclo, en nueve distritos preferentes: Arganzuela, Retiro, Salamanca, Chamartín, Tetuán, Chamberí, Fuencarral y Moncloa, en especial en Retiro, Salamanca, Chamberí y Chamartín, extendiéndose también a Ciudad Lineal. Coincide con la zona de estudios superiores de segundo ciclo, exceptuando el Centro, y prolongándose hacia el norte y este por Moncloa y Ciudad Lineal.

Los habitantes con estudios de formación profesional, como los más elevados, se extienden primordialmente en doce distritos: Centro, Arganzuela, Retiro, Salamanca, Chamartín, Tetuán, Chamberí, Fuencarral, Moncloa, Latina, Ciudad Lineal y Hortaleza, zona más amplia que la anterior, con diferencias menos acusadas, y que se extiende más hacia el nordeste y el sudoeste.

Los que poseen sólo el Bachillerato Superior se constriñen especialmente en su residencia a nueve distritos: Centro, Arganzuela, Retiro, Salamanca, Tetuán, Chamberí, Moncloa y Ciudad Lineal, coincidiendo con la zona de predominio de los estudios superiores de segundo ciclo, con la adición de Ciudad Lineal, aunque con valores muy dispares respecto a los demás distritos.

Los habitantes con Bachillerato Elemental se distribuyen por todos los distritos, si bien con valores superiores al promedio municipal figuran once de ellos: Centro, Arganzuela, Retiro, Salamanca, Chamartín, Tetuán, Chamberí, Moncloa, Latina, Ciudad Lineal y Hortaleza.

La población con estudios de enseñanza superior o de enseñanza media se halla ubicada primordialmente en los distritos interiores o centrales, aunque en ocasiones se ubica en algún distrito periférico.

Por el contrario, la población con sólo enseñanza primaria completa está muy generalizada, con promedio superior al municipal 
en once distritos, tres de ellos interiores -Centro, Arganzuela y Tetuán-y los demás - Latina, Carabanchel, Villaverde, Mediodía, Vallecas, Moratalaz, Ciudad Lineal y San Blas- periféricos.

La enseñanza primaria incompleta predomina en la población residente en nueve distritos, todos periféricos, salvo Fuencarral. La población analfabeta predomina en los mismos distritos que la enseñanza primaria incompleta.

\section{CARACTERISTICAS DE LA POBLACION ACTIVA}

\section{LA PROFESIÓN}

En la tabla VI se detalla la distribución de la población activa, según su profesión. Como es lógico, en una gran urbe la población con profesión del sector primario de la actividad económica es muy reducida. La población con profesiones propias del sector secundario vienen a representar una tercera parte de la población activa. Hay un evidente predominio de profesionales del sector de servicios.

Los profesionales liberales y técnicos residen con preferencia en todos los distritos centrales e interiores -en especial, Retiro, Salamanca, Chamartín y Chamberí- y también en los periféricos de Moncloa, Ciudad Lineal y Hortaleza.

El personal directivo, administrativo y asimilado de la Administración pública y de las empresas, reside preferentemente en casi todos los distritos centrales o interiores y en algunos periféricos, como Moncloa, Latina, Ciudad Lineal y Hortaleza.

Los comerciantes y vendedores están más extendidos entre todos los distritos, con diferencias menos acusadas de unos a otros, destacando tanto los centrales - Centro sobre todo, Arganzuela, Retiro y Tetuán- como algunos periféricos - Latina, Carabanchel, Villaverde, Moratalaz y Ciudad Lineal-. Distribución muy similar ofrece el personal de servicios, si bien sobresalen en los distritos interiores de Centro, Salamanca, Chamartín, Tetuán, Fuencarral y Chamberí, y en los periféricos de Moncloa y Vallecas.

Los habitantes con oficios diversos de la industria y transporte y peones no agrarios residen en forma muy destacada en todos los distritos periféricos, a excepción de Ciudad Lineal, además de en Fuencarral. 


\section{LA SITUACIÓN PROFESIONAL}

Según se desprende de la tabla VII, de cada 100 personas que forman parte de la población activa de Madrid, 85 trabajan a sueldo, jornal, comisión u otras formas de remuneración; cuatro son patronos, empresarios o profesionales; cinco, empresarios o profesionales que no emplean personal, trabajadores por cuenta propia o miembros de cooperativas; uno, trabajador familiar no remunerado, y el cinco restante no está debidamente clasificado.

El grupo más numeroso de los descritos se distribuye, con valores no muy dispares, entre todos los distritos municipales, si bien, como de residencia preferente, Retiro y Fuencarral, entre los distritos interiores, y todos los periféricos, a excepción de Moncloa y Ciudad Lineal.

Los patronos, empresarios y profesionales residen primordialmente en los distritos interiores - Centro, Retiro, Salamanca, Chamartín, Tetuán y Chamberí- y en los periféricos de Moncloa y Ciudad Lineal.

De análoga manera se distribuyen los empresarios o profesionales sin personal y los trabajadores por cuenta propia, residentes principalmente en los distritos de Centro, Arganzuela, Retiro, Salamanca, Chamartín, Tetuán y Chamberí, y en los periféricos de Moncloa, Carabanchel y Ciudad Lineal.

Los trabajadores familiares no remunerados se ubican especialmente en los distritos interiores - Chamberí con el valor máximoy en Moncloa.

\section{LA RAMA DE ACTIVIDAD ECONOMICA}

La tabla VIII complementa y amplía en cierto modo la tabla VI, de profesión. Agrupando las ramas de actividad por sectores económicos, se ofrece esta concreción de los habitantes que componen la población activa:

Porcentajes

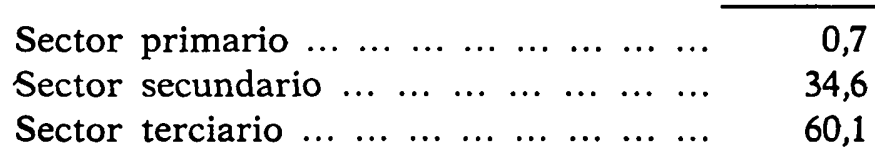

sin tener en cuenta las actividades no bien especificadas. 
La actividad agraria y de pesca y la explotación de minas y canteras es casi inexistente.

A industrias manufactureras se dedica casi la cuarta parte de la población activa, proporción que se eleva a más de la tercera parte si consideramos también las industrias de la energía y de la construcción. La población dedicada a industrias manufactureras y construcción reside de manera predominante en los distritos periféricos, mientras que la dedicada a energía predomina en muy pocos distritos: Arganzuela, Retiro y Tetuán entre los interiores, y Latina, Carabanchel y Moratalaz entre los periféricos.

En cuanto al sector de servicios, el personal de comercio y hostelería reside, ante todo, en algunos distritos interiores, como Centro, Arganzuela y Tetuán, y en otros periféricos, tales como Latina, Carabanchel, Vallecas, Moratalaz y Ciudad Lineal.

El personal de transporte, almacenamiento y comunicaciones reside preferentemente en distritos municipales periféricos $\mathrm{y}$, además, en alguno interior, como Arganzuela y Fuencarral.

La población activa dedicada a prestar sus servicios en establecimientos financieros, seguros y servicios prestados a las empresas, reside con preferencia en distritos interiores o centrales, y sólo excepcionalmente en algunos periféricos, como Moncloa, Latina, Ciudad Lineal y Hortaleza.

$\mathrm{Y}$, finalmente, el personal de servicios comunales, sociales $\mathrm{y}$ personales se ubica, sobre todo, en los distritos centrales o interiores y en alguno periférico, como Moncloa.

Expuestas en las líneas anteriores algunas conclusiones a las que nos ha permitido llegar el somero comentario de las cifras más importantes contenidas en las tablas transcritas en el apéndice estadístico, no se agotan por ello las posibilidades de análisis más profundos, utilizando dicha información y la contenida en la monografía que hemos contemplado.

Sería, de otra parte, muy interesante poder establecer una comparación entre las dos grandes ciudades españolas, Madrid y Barcelona, basada en el estudio de las características sobre las que se dispone de información estadística, al objeto de establecer las analogías y diferencias que se presentan en su conjunto y en el detalle de sus respectivos distritos municipales. 
T A B L A I

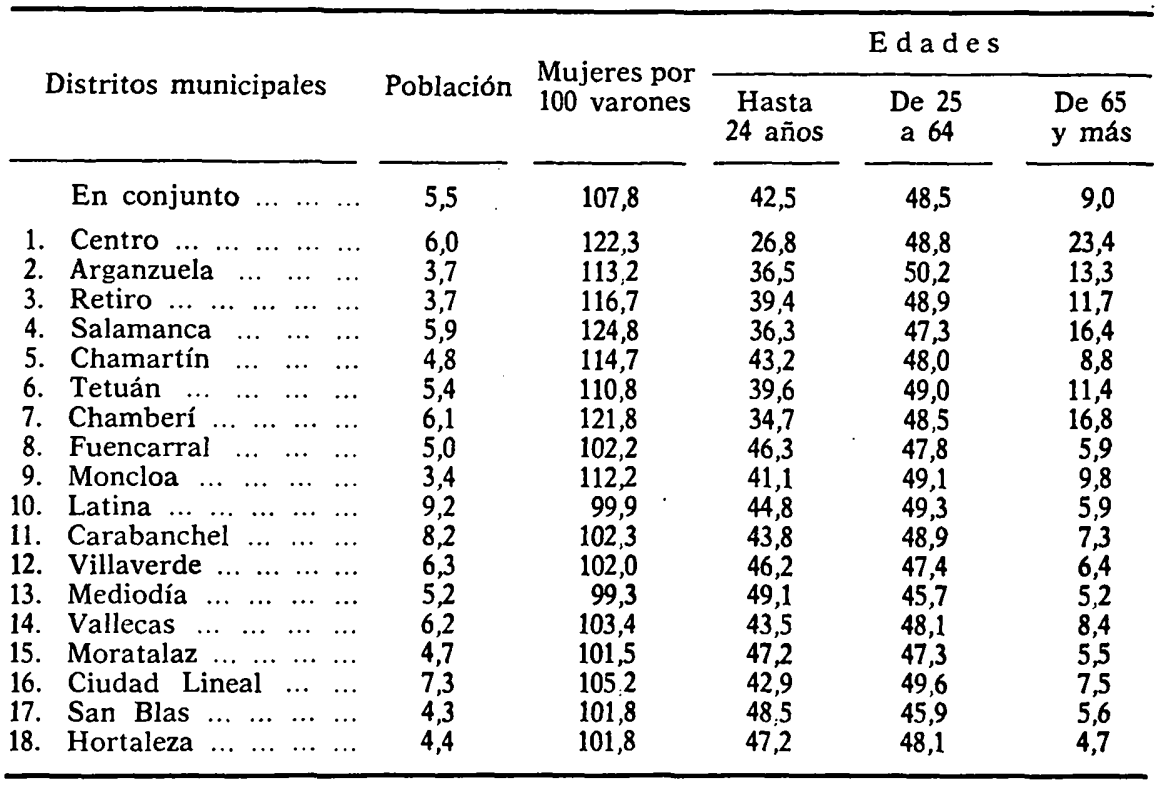

T A B L A I I

\section{LUGAR DE NACIMIENTO}

\begin{tabular}{|c|c|c|c|c|}
\hline \multicolumn{2}{|r|}{ Distritos municipales } & $\begin{array}{c}\text { Nacidos } \\
\text { en la Provincia } \\
\text { de Madrid }\end{array}$ & $\begin{array}{l}\text { Nacidos } \\
\text { en otras } \\
\text { Provincias }\end{array}$ & $\begin{array}{l}\text { Nacidos } \\
\text { en el } \\
\text { extranjero }\end{array}$ \\
\hline & & \multicolumn{3}{|c|}{ Porcentajes } \\
\hline & 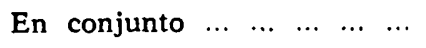 & 51,9 & 46,8 & 1,3 \\
\hline $\begin{array}{l}1 . \\
2 . \\
3 . \\
4 . \\
5 . \\
6 . \\
7 . \\
8 . \\
9 . \\
10 . \\
11 . \\
12 . \\
13 . \\
14 . \\
15 . \\
16 . \\
17 . \\
18 .\end{array}$ & 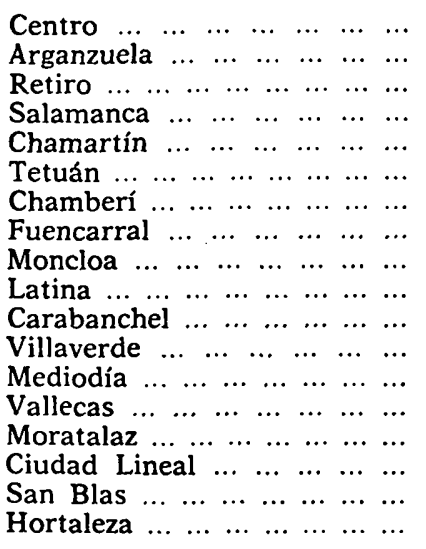 & $\begin{array}{l}52,2 \\
48,6 \\
52,1 \\
49,4 \\
53,2 \\
55,7 \\
49,8 \\
53,0 \\
50,3 \\
52,2 \\
50,2 \\
50,9 \\
50,4 \\
49,9 \\
53,5 \\
52,2 \\
57,5 \\
56,0\end{array}$ & $\begin{array}{l}46,9 \\
56,2 \\
46,2 \\
48,5 \\
44,3 \\
43,2 \\
48,6 \\
45,7 \\
47,9 \\
46,7 \\
48,8 \\
48,4 \\
48,9 \\
48,9 \\
45,2 \\
46,6 \\
41,6 \\
42,6\end{array}$ & $\begin{array}{l}0,9 \\
1,2 \\
1,7 \\
2,1 \\
2,5 \\
1,1 \\
1,6 \\
1,3 \\
1,8 \\
1,1 \\
1,0 \\
0,7 \\
0,7 \\
1,2 \\
1,3 \\
1,2 \\
0,9 \\
1,4\end{array}$ \\
\hline
\end{tabular}




\section{LUGAR DE NACIMIENTO}

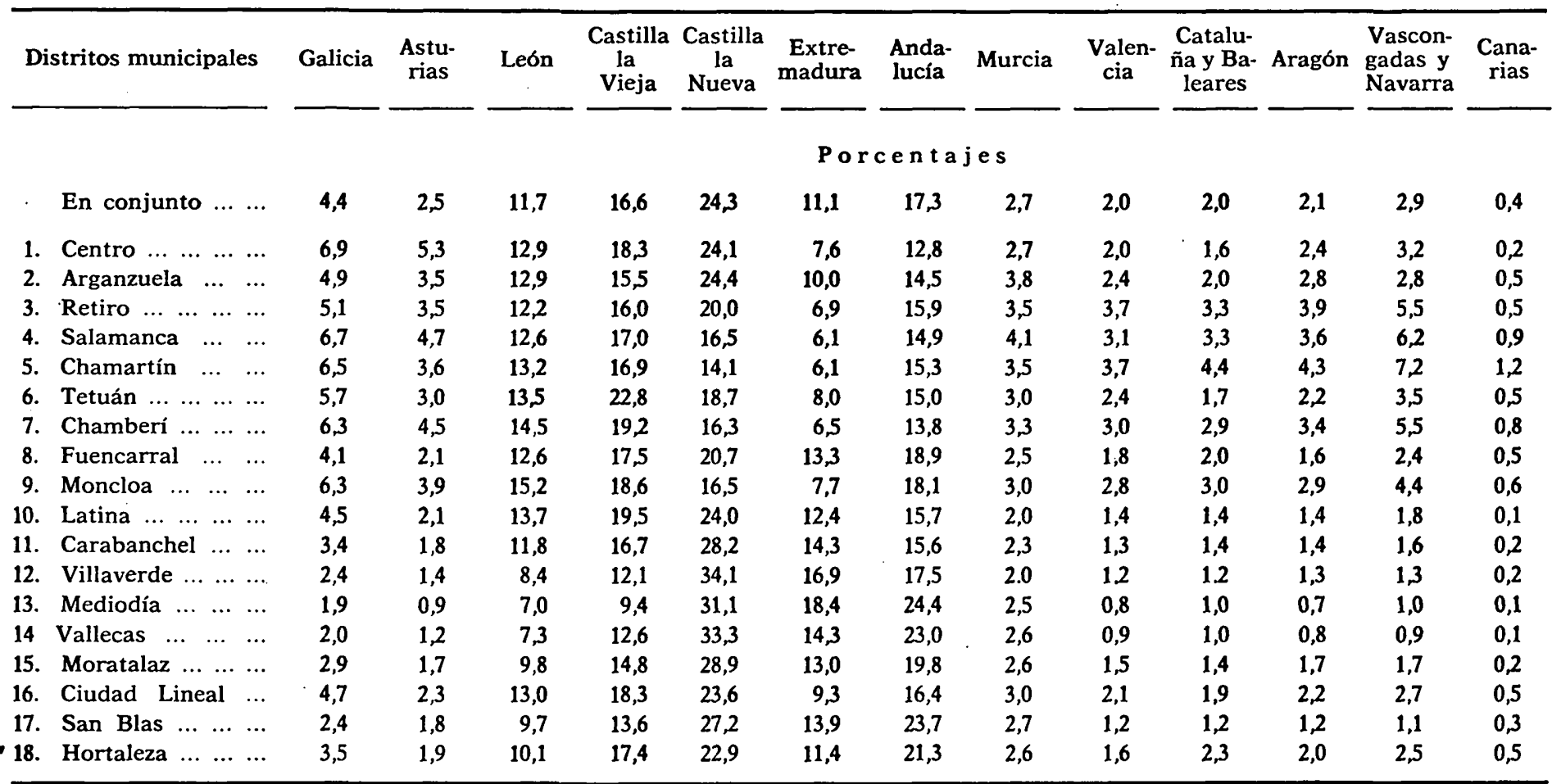


T A B L A I V

LUGAR DE NACIMIENTO

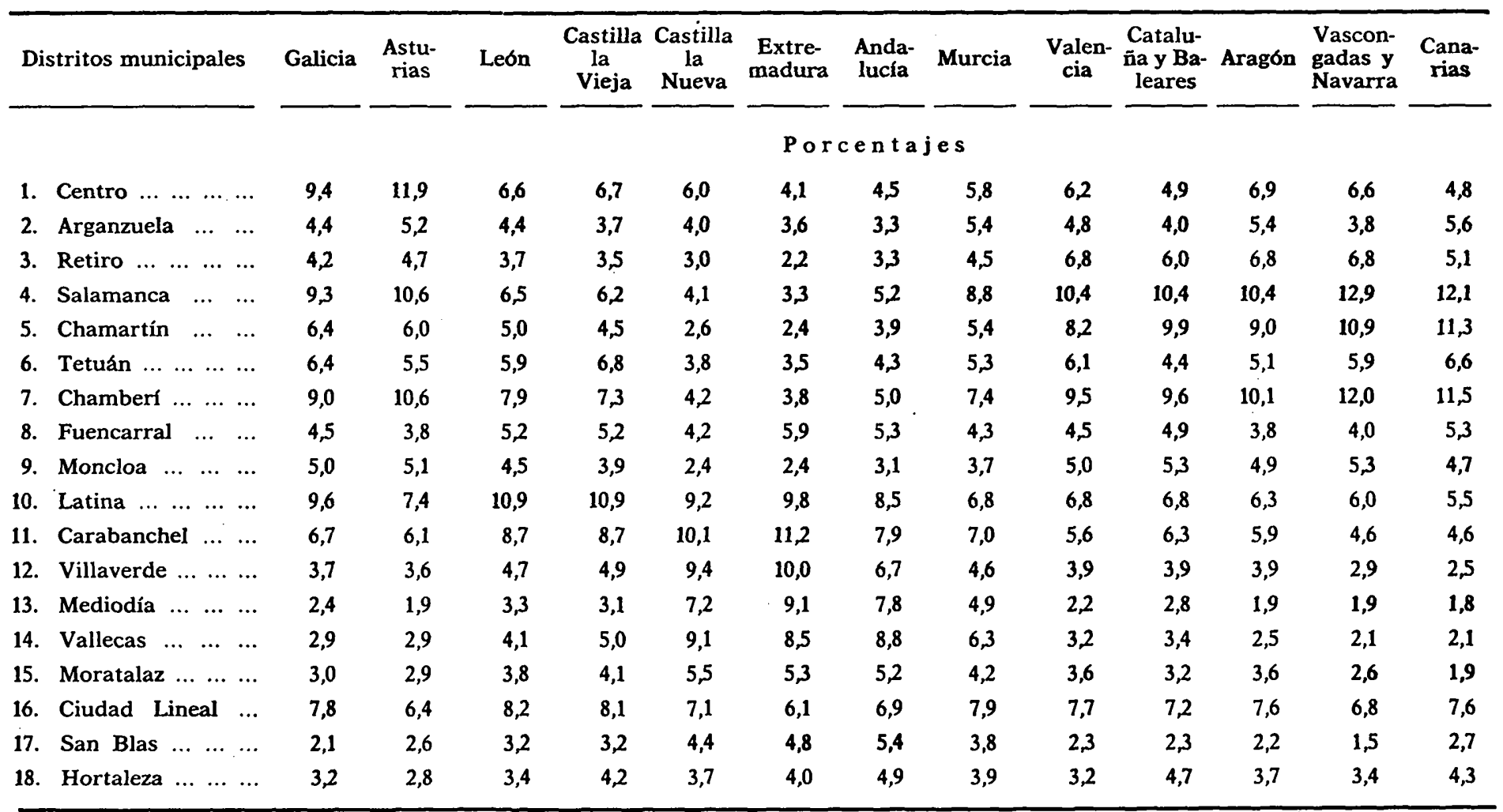

REVL-1979, núm. 204. BALLESTER ROS, IGNACIO. PRINCIPALES CARACTERISTICAS DE LA P... 
T A B L A V

CLASE DE ENSENANZA MAS ELEVADA RECIBIDA

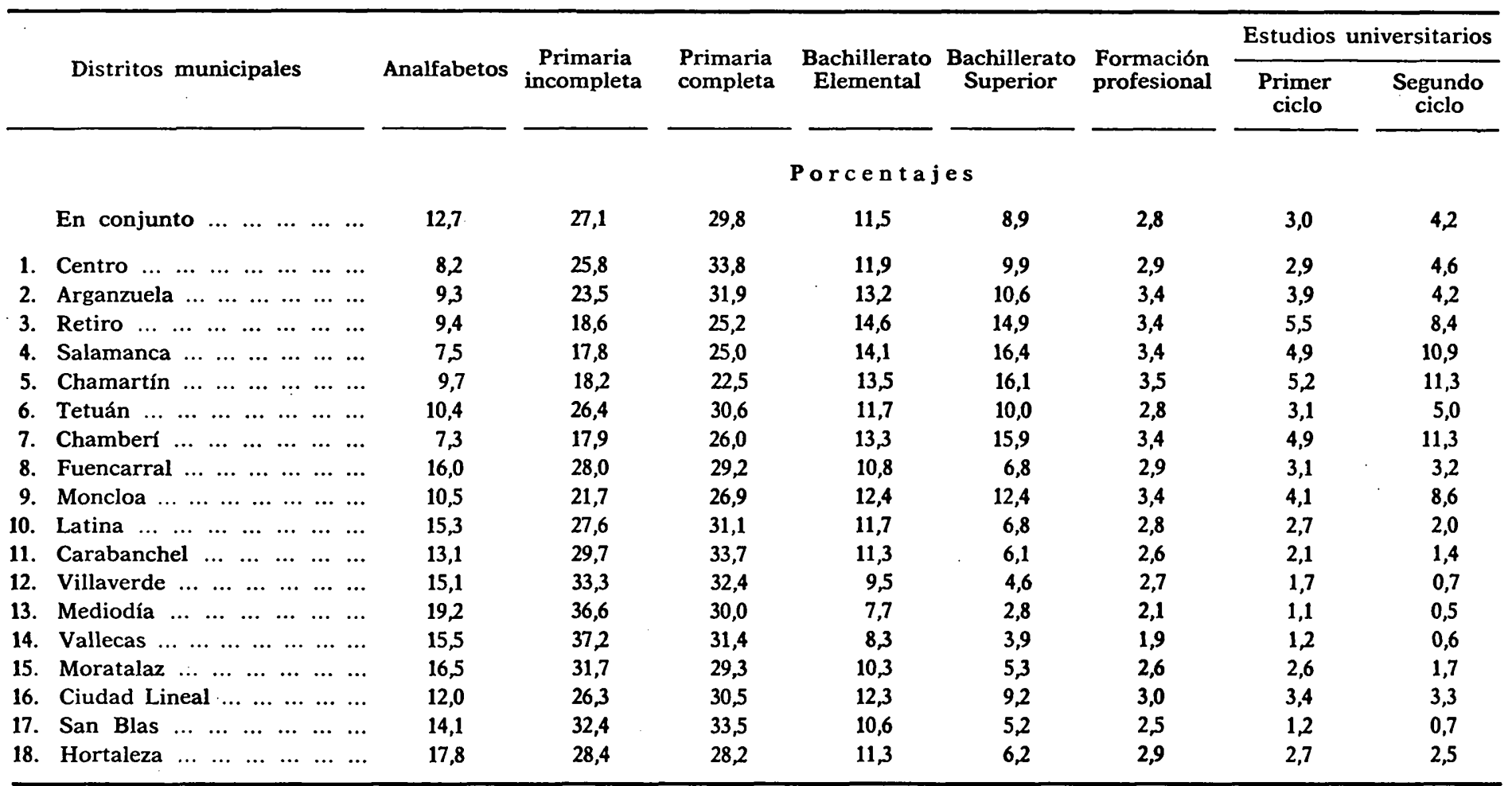




\section{TABLA VI}

\section{PROFESION}

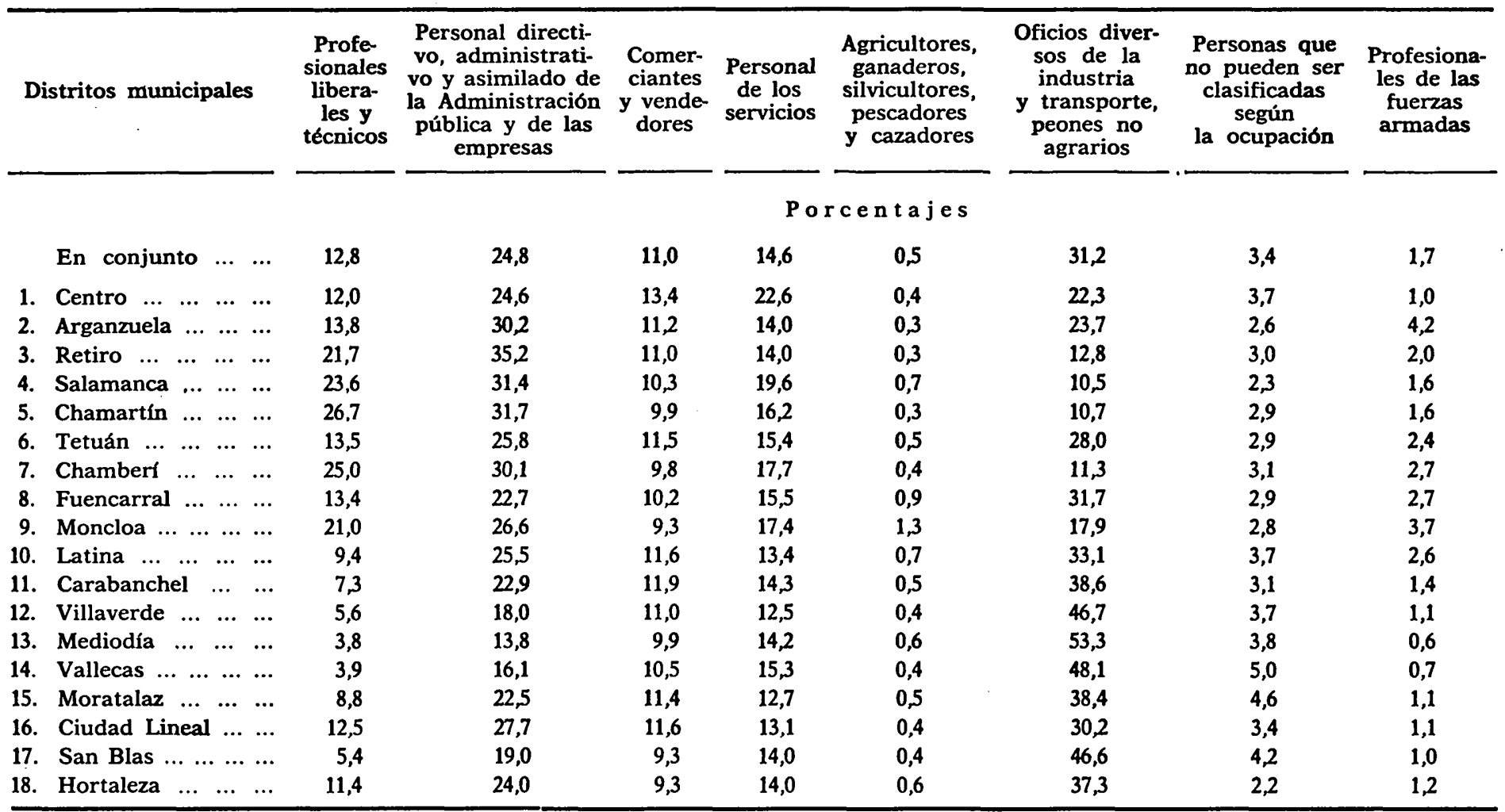

REVL-1979, núm. 204. BALLESTER ROS, IGNACIO. PRINCIPALES CARACTERISTICAS DE LA P... 
T A B L A V I I

\section{SITUACION PROFESIONAL}

\begin{tabular}{|c|c|c|c|c|c|c|}
\hline \multicolumn{2}{|r|}{ Distritos municipales } & $\begin{array}{l}\text { Patronos, } \\
\text { empresa- } \\
\text { rios y pro- } \\
\text { fesionales }\end{array}$ & $\begin{array}{l}\text { Empresarios o profesio- } \\
\text { nales que no emplean } \\
\text { personal, trabajadores } \\
\text { por cuenta propia } \\
\text { y miembros de } \\
\text { cooperativas }\end{array}$ & $\begin{array}{l}\text { Personas que traba- } \\
\text { jan a sueldo, jornal, } \\
\text { comisión o cualquier } \\
\text { otra forma de } \\
\text { remuneración }\end{array}$ & $\begin{array}{l}\text { Trabajadores, } \\
\text { familiares no } \\
\text { remunerados }\end{array}$ & $\begin{array}{l}\text { Población } \\
\text { activa no } \\
\text { clasificable en } \\
\text { otros grupos }\end{array}$ \\
\hline & & & & Porcentajes & & \\
\hline & En conjunto $\ldots \ldots \ldots \ldots \ldots$ & 3,9 & 5,1 & 84,8 & 1,2 & 5,0 \\
\hline 1. & 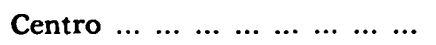 & 4,8 & 7,1 & 81,1 & 1,9 & 4,9 \\
\hline 2. & $\begin{array}{lllllll}\text { Arganzuela } & \ldots & \ldots & \ldots & \ldots & \ldots & \ldots\end{array}$ & 3,6 & 5,7 & 85,5 & 1,3 & 4,0 \\
\hline 3. & Retiro $\ldots \ldots \ldots \ldots \ldots$ & 5,9 & 5,6 & 83,6 & 1,2 & 3,7 \\
\hline 4. & $\begin{array}{lllllll}\text { Salamanca } & \ldots & \ldots & \ldots & \ldots & \ldots & \ldots\end{array}$ & 6,2 & 6,4 & 81,6 & 1,7 & 4,1 \\
\hline 5. & $\begin{array}{llllllll}\text { Chamartín } & \ldots & \ldots & \ldots & \ldots & \ldots & \ldots\end{array}$ & 7,4 & 5,5 & 80,7 & 1,8 & 4,6 \\
\hline 6. & Tetuán $\ldots \ldots \ldots \ldots \ldots$ & 4,7 & 5,9 & 83,9 & 1,4 & 4,1 \\
\hline 7. & 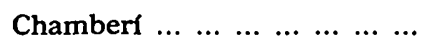 & 6,1 & 5,8 & 80,5 & 2,3 & 5,3 \\
\hline 8. & $\begin{array}{llllllll}\text { Fuencarral } & \ldots & \ldots & \ldots & \ldots & \ldots & \ldots\end{array}$ & 3,1 & 4,8 & 86,7 & 1,1 & 4,3 \\
\hline 9. & $\begin{array}{llllllll}\text { Moncloa } & \ldots & \ldots & \ldots & \ldots & \ldots & \ldots & \ldots\end{array}$ & 5,5 & 5,3 & 82,8 & 1,9 & 4,5 \\
\hline 10. & $\begin{array}{llllllllll}\text { Latina } & \ldots & \ldots & \ldots & \ldots & \ldots & \ldots & \ldots & \ldots\end{array}$ & 2,7 & 4,6 & 86,6 & 1,0 & 5,1 \\
\hline 11. & Carabanchel $\ldots \ldots \ldots c c c c$ & 3,4 & 5,1 & 85,9 & 0,8 & 4,8 \\
\hline 12. & $\begin{array}{lllllll}\text { Villaverde } & \ldots & \ldots & \ldots & \ldots & \ldots & \ldots\end{array}$ & 2,9 & 4,2 & 86,0 & 1,0 & 5,9 \\
\hline & Mediodía $\ldots \ldots \ldots \ldots$ & 1,8 & 3,8 & 87,6 & 0,7 & 6,1 \\
\hline & $\begin{array}{llllllll}\text { Vallecas } & \ldots & \ldots & \ldots & \ldots & \ldots & \ldots & \ldots\end{array}$ & 2,2 & 4,5 & 87,4 & 0,6 & 5,3 \\
\hline & $\begin{array}{lllllll}\text { Moratalaz } & \ldots & \ldots & \ldots & \ldots & \ldots & \ldots\end{array}$ & 2,8 & 4,4 & 86,4 & 0,7 & 5,7 \\
\hline & 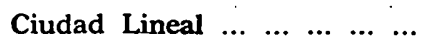 & 4,1 & 5,7 & 84,0 & 1,1 & 5,1 \\
\hline & 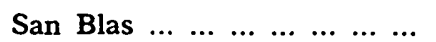 & 1,9 & 3,7 & 87,7 & 0,6 & 6,1 \\
\hline & 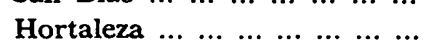 & 2,9 & 4,1 & 87,9 & 0,9 & 4,2 \\
\hline
\end{tabular}




\section{T A B L A V I I I}

RAMA DE ACTIVIDAD

\begin{tabular}{|c|c|c|c|c|c|c|c|c|c|c|c|}
\hline & $\begin{array}{c}\text { Distritos } \\
\text { municipales }\end{array}$ & $\begin{array}{l}\text { Agricultu- } \\
\text { ra, ganade- } \\
\text { ría, silvi- } \\
\text { cultura } \\
\text { y pesca }\end{array}$ & $\begin{array}{l}\text { Explota- } \\
\text { ción de } \\
\text { minas y } \\
\text { canteras }\end{array}$ & $\begin{array}{l}\text { Indus- } \\
\text { trias } \\
\text { manufac- } \\
\text { tureras }\end{array}$ & $\begin{array}{l}\text { Electri- } \\
\text { cidad, } \\
\text { gas } \\
\text { y agua }\end{array}$ & $\begin{array}{l}\text { Cons- } \\
\text { truc- } \\
\text { ción }\end{array}$ & $\begin{array}{l}\text { Comercio } \\
\text { al por ma- } \\
\text { yor, restau- } \\
\text { rantes y } \\
\text { hoteles }\end{array}$ & $\begin{array}{l}\text { Transpor- } \\
\text { tes, almace- } \\
\text { namientos } \\
\text { y comuni- } \\
\text { caciones }\end{array}$ & $\begin{array}{l}\text { Establecimientos } \\
\text { financieros, segu- } \\
\text { ros, bienes in- } \\
\text { muebles y servi- } \\
\text { cios prestados a } \\
\text { las empresas }\end{array}$ & $\begin{array}{c}\text { Servi- } \\
\text { cios co- } \\
\text { munales, } \\
\text { sociales } \\
\text { y perso- } \\
\text { nales }\end{array}$ & $\begin{array}{l}\text { Activi- } \\
\text { dades } \\
\text { no bien } \\
\text { especifi- } \\
\text { cadas }\end{array}$ \\
\hline & & & \multicolumn{9}{|c|}{ Porcentajes } \\
\hline & En conjunto $\ldots$ & 0,6 & 0,1 & 24,3 & 0,7 & 9,6 & 15,9 & 8,0 & 8,6 & 27,6 & 4,6 \\
\hline 2. & Arganzuela ... ... & 0,5 & 0,1 & 23,6 & 0,7 & 5,6 & 17,7 & 9,1 & 9,3 & 29,7 & 3,7 \\
\hline 3. & $\begin{array}{llll}\text { Retiro } & \ldots & \ldots & \ldots\end{array}$ & 0,5 & 0,1 & 18,6 & 1,0 & 5,4 & 14,2 & 7,2 & 12,4 & 36,3 & 4,3 \\
\hline 4. & Salamanca ... ... & 0,9 & 0,2 & 15,4 & 0,6 & 5,0 & 13,7 & 5,4 & 11,9 & 43,2 & 3,7 \\
\hline 5. & Chamartín ... ... & 0,5 & 0,2 & 16,6 & 0,6 & 5,6 & 12,0 & 6,2 & 13,4 & 40,1 & 4,8 \\
\hline 6. & Tetuán $\ldots \ldots \ldots$ & 0,6 & 0,1 & 21,9 & 0,9 & 9,0 & 16,5 & 7,6 & 8,4 & 30,9 & 4,1 \\
\hline 7. & Chamberi $\ldots \quad \ldots$ & 0,5 & 0,2 & 13,5 & 0,6 & 4,5 & 13,4 & 5,4 & 10,6 & 46,8 & 4,5 \\
\hline 13. & Mediodía ... ... ... & 0,7 & 0,1 & 32,5 & 0,5 & 18,8 & 14,5 & 9,1 & 4,4 & 15,0 & 4,4 \\
\hline 14. & Vallecas ... ... ... & 0,5 & 0,1 & 29,1 & 0,4 & 17,0 & 16,5 & 7,8 & 5,5 & 17,6 & 5,5 \\
\hline 15. & Moratalaz $\ldots \ldots$ & 0,5 & 0,2 & 28,5 & 1,0 & 12,3 & 16,1 & 8,2 & 8,3 & 19,4 & 5,5 \\
\hline 16. & Ciudad Lineal ... & 0,5 & 0,2 & 26,0 & 0,6 & 8,6 & 16,1 & 9,1 & 9,4 & 24,9 & 4,6 \\
\hline 17. & San Blas $\ldots \ldots$ & 0,5 & 0,1 & 34,1 & 0,6 & 12,6 & 14,9 & 7,8 & 6,5 & 17,8 & 5,1 \\
\hline 18. & $\begin{array}{ccc}\text { Hortaleza } & \ldots & \ldots\end{array}$ & 0,7 & 0,1 & 28,0 & 0,6 & 11,7 & 14,3 & 10,5 & 9,4 & 21,3 & 3,4 \\
\hline
\end{tabular}

REVL-1979, núm. 204. BALLESTER ROS, IGNACIO. PRINCIPALES CARACTERISTICAS DE LA P... 


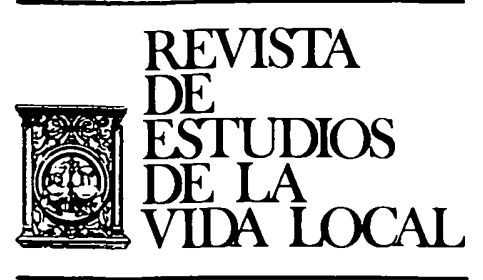

\section{JURISPRUDENCIA}


REVL-1979, núm. 204. BALLESTER ROS, IGNACIO. PRINCIPALES CARACTERISTICAS DE LA P... REVL-1979, núm. 204. BALLESTER ROS, IGNACIO. PRINCIPALES CARACTERISTICAS DE LA P... 